\title{
Achados à prova calórica e canal semicircular acometido na vertigem posicional paroxística benigna
}

\section{Caloric test results and damaged semicircular canal in benign positional paroxysmal vertigo}

\author{
Andréa Manso ${ }^{1}$, Cristina Freitas Ganança ${ }^{2}$, Fernando Freitas Ganança ${ }^{3}$, Maurício Malavasi Ganança ${ }^{4}$, \\ Heloisa Helena Caovilla ${ }^{5}$
}

\begin{abstract}
RESUMO
Objetivo: Caracterizar os achados da prova calórica em pacientes com vertigem posicional paroxística benigna, segundo o canal semicircular comprometido. Métodos: Foram analisados 1033 prontuários de pacientes submetidos à pesquisa de nistagmo posicional e de posicionamento e à eletronistagmografia. Os achados da prova calórica, de acordo com os canais semicirculares acometidos, foram submetidos à análise estatística. Resultados: No comprometimento de canal posterior, houve prevalência de normorreflexia $(p<0,0001)$; hiporreflexia prevaleceu sobre hiper-reflexia $(p<0,0001)$ e preponderância direcional $(p<0,0001)$, e hiper-reflexia prevaleceu sobre preponderância direcional $(\mathrm{p}<0,0001)$. No comprometimento de canal lateral, normorreflexia prevaleceu sobre hiporreflexia $(\mathrm{p}<0,0001)$ e hiper-reflexia $(\mathrm{p}<0,0001)$; hiporreflexia tendeu a prevalecer sobre hiper-reflexia $(\mathrm{p}=0,0771)$, e preponderância direcional não ocorreu. No comprometimento de canal anterior, normorreflexia prevaleceu sobre hiporreflexia $(\mathrm{p}<0,0001)$; hiper-reflexia e preponderância direcional não ocorreram. Conclusão: Na prova calórica de pacientes com vertigem posicional paroxística benigna, normorreflexia, hiporreflexia, hiper-reflexia ou preponderância direcional do nistagmo pós-calórico ocorrem em ordem decrescente de prevalência, quando há comprometimento do canal posterior; normorreflexia prevalece sobre hiporreflexia ou hiper-reflexia e hiporreflexia tende a prevalecer sobre hiper-reflexia, no comprometimento do canal lateral; normorreflexia prevalece sobre hiporreflexia, no comprometimento do canal anterior.
\end{abstract}

Descritores: Labirinto; Canais semicirculares; Nistagmo posicional; Tontura; Vertigem; Eletronistagmografia; Testes calóricos/ métodos

\section{INTRODUÇÃO}

Tontura é a sensação de perturbação do equilíbrio corporal e encontra-se entre os sintomas mais comuns no mundo, podendo ser desencadeadas por disfunção primária ou secundária do sistema vestibular. A disfunção vestibular pode ter locali-

Trabalho realizado nos Departamentos de Otorrinolaringologia, de Cirurgia de Cabeça e Pescoço e de Fonoaudiologia da Universidade Federal de São Paulo - UNIFESP - São Paulo (SP), Brasil, com auxílio à pesquisa fomentado pela Coordenação de Aperfeiçoamento de Pessoal de Nível Superior (CAPES).

(1) Mestre, Fonoaudióloga do Hospital Cema - São Paulo (SP), Brasil.

(2) Doutora, Professora Substituta do Departamento de Fonoaudiologia da Universidade Federal de São Paulo - UNIFESP - São Paulo (SP), Brasil.

(3) Doutor, Professor Adjunto da Disciplina de Otoneurologia da Universidade Federal de São Paulo - UNIFESP - São Paulo (SP), Brasil.

(4) Livre-docente, Professor Titular de Otorrinolaringologia da Universidade Federal de São Paulo - UNIFESP - São Paulo (SP), Brasil.

(5) Livre-docente, Professora Associada da Disciplina de Otoneurologia da Universidade Federal de São Paulo - UNIFESP - São Paulo (SP), Brasil.

Endereço para correspondência: Andréa Manso. R. Dr. César, 1227/121, Santana, São Paulo - SP, CEP: 02022-030.E-mail: andreamanso@ bol.com.br Recebido em: 9/11/2007; Aceito em: 7/10/2008 zação periférica ou central e sua causa pode estar situada em outro órgão ou sistema do corpo humano(1).

Entre as vestibulopatias periféricas, a vertigem posicional paroxística benigna (VPPB) é a mais frequente, muito comum em jovens, adultos e idosos sendo rara em crianças. A queixa característica é de tontura giratória à mudança de cabeça, ao olhar para cima, ao deitar-se para um lado ou para os dois lados ou levantar-se, podendo ser acompanhada de náuseas, vômitos e, entre as crises, pode ocorrer instabilidade intermitente ou outros tipos de tontura. $\mathrm{O}$ acometimento unilateral $(88,2 \%)$ é prevalente sobre bilateral $(11,9 \%)$ em pacientes com $\mathrm{VPPB}^{(2)}$.

A fisiopatologia da VPPB é explicada pela presença indevida de partículas de carbonato de cálcio provenientes de fracionamento de estatocônios da mácula utricular, na cúpula da crista ampular ou na endolinfa de um ou mais ductos semicirculares. Foi denominado de cupulolitíase o depósito destas partículas na cúpula do canal semicircular posterior ${ }^{(3)}$ e de canalitíase o processo de flutuação livre das partículas na endolinfa do canal semicircular posterio ${ }^{(4)}$ o termo ductolitíase também é utilizado ${ }^{(1)}$. 
A VPPB acomete com maior frequência o canal semicircular posterior, mas também pode envolver o canal lateral e o canal anterior ${ }^{(5)}$. Em 168 pacientes com VPPB, o acometimento de canal posterior ocorreu em $89,9 \%$ dos casos, do canal lateral em 8,3\% e do anterior em 1,8\% ${ }^{(6)}$. Em 122 casos de VPPB, $110(90,2 \%)$ apresentaram comprometimento de canal posterior, $10(8,2 \%)$ de canal lateral e dois $(1,6 \%)$ de canal anterior ${ }^{(7)}$. A prevalência de acometimento do canal lateral foi observada em $16,4 \%$ dos casos de $\mathrm{VPPB}^{(8)}$.

Para estabelecer o diagnóstico de VPPB, foi elaborada uma prova para avaliar a vertigem e o nistagmo de posicionamento, no qual o paciente, da posição sentada, com a cabeça voltada 45 graus para o lado a ser avaliado, passa rapidamente para a posição de cabeça pendente e inclinada 45 graus para o lado avaliado, por aproximadamente 30 segundos, com a ajuda do examinador. Após a extinção do nistagmo de posicionamento, o paciente retorna à posição sentada, sendo a manobra repetida para o outro lado $^{(9)}$.

A pesquisa do nistagmo de posicionamento, por meio da observação da direção e duração do nistagmo, possibilita a identificação do lado lesado e do canal semicircular acometido $^{(10)}$.

Nos pacientes vertiginosos, o comprometimento do sistema vestibular pode ser aferido por meio da avaliação otoneurológica, que pode localizar o lado da lesão, ajuizar a intensidade de comprometimento, estabelecer o prognóstico e orientar o tratamento $^{(11)}$.

$\mathrm{Na}$ VPPB, os testes convencionais de avaliação do reflexo vestíbulo-ocular e a eletronistagmografia geralmente não mostram alterações típicas, com exceção dos procedimentos de pesquisa da vertigem e nistagmo de posicionamento e/ ou posicional. No entanto, a eletronistagmografia é capaz de identificar sinais de disfunção vestibular em alguns $\operatorname{casos}^{(6-12)}$. A prova calórica costuma ser normal na maioria dos pacientes com VPPB ${ }^{(13-14)}$. À prova calórica de 168 pacientes com VPPB foi identificada normorreflexia em $57,2 \%$ de e anormalidades do nistagmo pós-calórico em 72 casos $(42,8 \%)$ : hiporreflexia unilateral em 22,0\%, preponderância direcional em 13,7\% e hiporreflexia unilateral concomitante com preponderância direcional em 7,1\%; destacando que as alterações à eletronistagmografia são comuns na VPPB de canal posterior ${ }^{(6)}$. Hiporreflexia do nistagmo pós-calórico ocorreu em 16,6\% de 18 pacientes com $\mathrm{VPPB}^{(15)}$.

Na VPPB de canal posterior, a normorreflexia foi identificada em $51,7 \%$ de 240 casos, como achado predominante na prova calórica, a hiporreflexia ocorreu em cerca de $39,0 \%$ dos casos e a preponderância direcional em cerca de $8 \%$; sugerindo que os pacientes com hiporreflexia do nistagmo pós-calórico ipsilateral ao labirinto comprometido pela VPPB apresentariam envolvimento simultâneo do canal semicircular lateral e posterior do mesmo lado ${ }^{(16)}$. Foram encontradas normorreflexia em $46,0 \%$ de 45 casos, hiporreflexia ipsilateral em 16,0\%, hiporreflexia contralateral em 7,0\%, hiporreflexia bilateral em $8,0 \%$ e preponderância direcional em $8,0 \%{ }^{(17)}$.

Em 20 pacientes com VPPB de canal posterior unilateral, 45,0\% dos casos apresentaram hiporreflexia ipsilateral, 30,0\% normorreflexia, $15,0 \%$ hiporreflexia contralateral e 5,0\% preponderância direcional; a presença de hiporreflexia nestes pacientes sugere uma lesão ou hipofunção concomitante do canal lateral ${ }^{(18)}$.

Hiporreflexia do nistagmo pós-calórico ipsilateral ao canal lateral comprometido pela VPPB foi observada em $33,3 \%$ de $15 \operatorname{casos}^{(19)}$, em $30,8 \%$ de $13 \operatorname{casos}^{(20)}$, em um paciente ${ }^{(21)}$ e em cerca de $30,0 \%$ dos $\operatorname{casos}^{(22)}$. A hiporreflexia do nistagmo póscalórico ipsilateral ao canal lateral afetado pela VPPB seria devida a uma obstrução parcial do canal lateral; as manobras liberatórias normalizaram os resultados da prova calórica ${ }^{(22)}$. Em 16 casos de VPPB de canal lateral, a hiporreflexia do nistagmo pós-calórico ipsilateral ocorreu em $50,0 \%$, e respostas simétricas foram observadas à prova calórica após as manobras liberatórias ${ }^{(23)}$.

Em 122 casos de VPPB, normorreflexia do nistagmo póscalórico foi encontrada em 58 pacientes $(47,6 \%)$; alterações do nistagmo pós-calórico foram reconhecidas em 64 pacientes $(52,4 \%)$ : hiporreflexia em $27,0 \%$ dos casos (unilateral em $13,9 \%$ e bilateral em $13,1 \%$ ), preponderância direcional em $17,2 \%$ e preponderância direcional concomitante com hiporreflexia $(8,2 \%)$. Nos 110 casos de VPPB de canal posterior, a hiporreflexia foi identificada em $26,4 \%$ dos casos (unilateral em $13,7 \%$ e bilateral em $12,7 \%$ ), preponderância direcional em $18,2 \%$ e hiporreflexia concomitante com preponderância direcional em 5,5\%. Na VPPB de canal posterior unilateral, a hiporreflexia ipsilateral foi evidenciada em $8,2 \%$ dos casos e a contralateral em 5,5\%. Nos 10 casos de VPPB de canal lateral, hiporreflexia à prova calórica foi encontrada em dois casos (ipsilateral em um e bilateral em outro), preponderância direcional em um caso, hiporreflexia ipsilateral concomitante com preponderância direcional em quatro casos. Na prova calórica dos dois casos de VPPB de canal anterior, a hiporreflexia foi ipsilateral em um deles e bilateral no outro ${ }^{(7)}$.

O nistagmo pós-calórico foi avaliado em 68 pacientes: $41,2 \%$ dos casos apresentaram sinais sugestivos de síndrome vestibular periférica irritativa, 30,9\% hiporreflexia e $27,9 \%$ normorreflexia ${ }^{(24)}$.

A avaliação vestibular completa na VPPB é importante porque foi observada que a hiporreflexia unilateral em pacientes com história clínica de outra doença otológica prévia é mais frequente do que naqueles sem sintomas de doença otológica anterior ${ }^{(25)}$.

Esta investigação foi motivada pelo interesse na avaliação de anormalidades do nistagmo pós-calórico ipsilateral ou contralateral ao canal semicircular comprometido pela VPPB. A análise conjunta de achados da prova calórica e da pesquisa do nistagmo de posicionamento e posicional poderia contribuir para melhor caracterização da VPPB, com possíveis implicações terapêuticas e prognósticas em cada paciente.

O objetivo deste estudo foi caracterizar os achados na prova calórica em pacientes com VPPB de acordo com o canal semicircular comprometido.

\section{MÉTODOS}

Esta pesquisa foi aprovada pelo Comitê de Ética em Pesquisa da Universidade Federal de São Paulo - UNIFESP, sob protocolo de número 1381/06.

Para a seleção da casuística foram analisados os prontuá- 
rios dos pacientes atendidos de janeiro de 1999 a julho de 2004, independentemente da idade e sexo. O diagnóstico foi estabelecido pelo otorrinolaringologista de VPPB, com base na história clínica e nos achados à pesquisa do nistagmo de posicionamento e/ou posicional.

Foram incluídos nesta pesquisa pacientes com relato de episódios de tontura rotatória à mudança da posição da cabeça, ao deitar-se para um lado ou para os dois lados, ao levantar-se ou ao olhar para cima, com ou sem náuseas e vômitos e presença de nistagmo de posicionamento e ou posicional. Foram excluídos os pacientes que apresentaram sinais de comprometimento do sistema nervoso central ou outra vestibulopatia concomitante.

Os participantes desta pesquisa foram submetidos à pesquisa de nistagmo e/ou vertigem de posicionamento à prova de Dix-Hallpike, realizadas com lentes de Frenzel (Neurograff Eletromedicina Ind. e Com. Ltda - EPT - Brasil), e à pesquisa de nistagmo posicional, nos decúbitos laterais.

As características do nistagmo de posicionamento e/ ou posicional determinaram o labirinto acometido e o canal semicircular afetado: nistagmo vertical para cima e rotatório caracterizou o comprometimento do canal posterior; nistagmo vertical para baixo e rotatório caracterizou o comprometimento do canal anterior; nistagmo rotatório indicou o comprometimento de canal vertical, sem distinguir entre canal posterior e anterior, e nistagmo horizontal apontou o comprometimento de canal lateral.

Também foram submetidos a testes que avaliam a função vestibular por meio de electronistagmografia computadorizada (Micromedial Technologies, Inc.), e otocalorímetro a ar (Neurograff Eletromedicina Ind. e Com. Ltda - EPT Brasil) composta pela pesquisa de nistagmo espontâneo e semi-espontâneo, movimentos sacádicos, rastreio pendular, nistagmo optocinético, auto-rotação cefálica e prova calórica com ar a $42^{\circ}$ e $18^{\circ} \mathrm{C}$. Os testes utilizados foram realizados e interpretados de acordo com critérios de normalidade descritos na literatura ${ }^{(26)}$.

Os dados foram submetidos à análise estatística. Foi utilizada análise descritiva simples dos dados por frequências absolutas e porcentagens para caracterização dos canais semicirculares acometidos em resposta aos achados da prova calórica, normorreflexia, hiporreflexia, hiper-reflexia ou preponderância direcional.

Foi empregado o teste Qui-quadrado de Aderência na análise inferencial dos dados, para verificar associação entre as frequências de acometimento dos canais semicirculares. $\mathrm{O}$ teste Qui-quadrado de Aderência, seguido pela correção de Yates, foi empregado na análise de associação entre as frequências aos pares dos canais comprometidos. Foi utilizado o teste Qui-quadrado $\left(\chi^{2}\right)$ para verificar a associação entre canal semicircular e a prova calórica. Quando verificada diferença entre os resultados $(p<0,05)$, foi aplicada partição da tabela para comparação múltipla dos pares, verificando os pares de grupos com diferenças. Foi também realizada a análise para comparação intragrupo dos resultados da prova calórica. Os testes foram aplicados com nível de significância de 5\% $(\alpha=0,05)$.

\section{RESULTADOS}

Foram analisados os prontuários de 1033 pacientes com hipótese diagnóstica de VPPB, com base na história clínica e nos achados à pesquisa do nistagmo de posicionamento e/ ou posicional.

A Tabela 1 apresenta o número e a porcentagem de casos, de acordo com o canal semicircular comprometido uni ou bilateralmente na VPPB.

O comprometimento do canal posterior prevaleceu sobre o dos outros canais $(\mathrm{p}<0,0001)$. O acometimento unilateral do canal posterior predominou sobre o bilateral $(\mathrm{p}<0,0001)$. O comprometimento do canal lateral prevaleceu sobre o do canal anterior ( $\mathrm{p}=0,0002)$. Não houve ocorrência de acometimento bilateral dos canais anterior e lateral.

A Tabela 2 apresenta o número e a porcentagem de casos com normorreflexia, hiporreflexia, hiper-reflexia ou preponderância direcional do nistagmo pós-calórico, de acordo com o canal semicircular comprometido na VPPB.

O canal posterior apresentou prevalência de normorreflexia em comparação com os demais achados anormais à prova calórica $(\mathrm{p}<0,0001)$; a hiporreflexia foi prevalente sobre a hiper-reflexia $(p<0,0001)$ e a preponderância direcional do nistagmo pós-calórico ( $\mathrm{p}<0,0001)$, e a hiper-reflexia foi prevalente sobre a preponderância direcional do nistagmo pós-calórico $(\mathrm{p}<0,0001)$.

O canal lateral apresentou prevalência de normorreflexia em comparação com hiporreflexia $(\mathrm{p}<0,0001)$ e hiper-reflexia $(\mathrm{p}<0,0001)$; a hiporreflexia apresentou tendência de prevalência sobre a hiper-reflexia $(\mathrm{p}=0,0771)$, e a preponderância direcional do nistagmo pós-calórico não foi identificada.

Tabela 1. Frequências absolutas, porcentagens e análise comparativa do comprometimento uni ou bilateral dos canais semicirculares em 1033 pacientes com vertigem posicional paroxística benigna

\begin{tabular}{|c|c|c|c|c|c|c|c|}
\hline \multirow{3}{*}{ Canal } & \multicolumn{6}{|c|}{ Comprometimento } & \multirow{3}{*}{$\begin{array}{l}\text { Qui-quadrado } \\
\text { (Valor de p) }\end{array}$} \\
\hline & \multicolumn{2}{|c|}{ Unilateral } & \multicolumn{2}{|l|}{ Bilateral } & \multicolumn{2}{|l|}{ Total } & \\
\hline & $\mathrm{N}$ & $\%$ & $\mathrm{~N}$ & $\%$ & $\mathrm{~N}$ & $\%$ & \\
\hline \multirow{4}{*}{$\begin{array}{l}\text { Posterior } \\
\text { Lateral } \\
\text { Anterior }\end{array}$} & 814 & 78,8 & 85 & 8,2 & 899 & 87,0 & \multirow{4}{*}{$\begin{array}{l}<0,0001 \\
\text { Posterior }- \text { Lateral }<0,0001 \\
\text { Posterior }- \text { Anterior }<0,0001 \\
\text { Lateral }- \text { Anterior }=0,0002\end{array}$} \\
\hline & 89 & 8,6 & -- & -- & 89 & 8.6 & \\
\hline & 45 & 4,4 & -- & -- & 45 & 4.4 & \\
\hline & & & & & & & \\
\hline Total & 948 & 91,8 & 85 & 8,2 & 1.033 & 100,0 & \\
\hline
\end{tabular}

Nível de significância: $p \leq 0,05$ 
Tabela 2. Frequências absolutas, porcentagens e análise comparativa dos achados à prova calórica de acordo com os canais semicirculares comprometidos dos 1033 pacientes com vertigem posicional paroxística benigna

\begin{tabular}{|c|c|c|c|c|c|c|c|c|c|c|}
\hline \multirow{3}{*}{ Canal } & \multicolumn{8}{|c|}{ Achados à prova calórica } & \multirow{3}{*}{\multicolumn{2}{|c|}{$\begin{array}{l}\text { Qui-quadrado } \\
\text { (valor de p) }\end{array}$}} \\
\hline & \multicolumn{2}{|c|}{ Normorreflexia } & \multicolumn{2}{|c|}{ Hiporreflexia } & \multicolumn{2}{|c|}{ Hiper-reflexia } & \multicolumn{2}{|c|}{ PDN } & & \\
\hline & $\mathrm{N}$ & $\%$ & $\mathrm{~N}$ & $\%$ & $\mathrm{~N}$ & $\%$ & $\mathrm{~N}$ & $\%$ & & \\
\hline \multirow{6}{*}{ Posterior } & \multirow{6}{*}{723} & \multirow{6}{*}{80,4} & \multirow{6}{*}{126} & \multirow{6}{*}{14,0} & \multirow{6}{*}{41} & \multirow{6}{*}{4,6} & \multirow{6}{*}{9} & \multirow{6}{*}{1,0} & \multirow{6}{*}{$<0,0001$} & N - Нipo <0,0001 \\
\hline & & & & & & & & & & N - Hiper <0,0001 \\
\hline & & & & & & & & & & $N-P D N<0,0001$ \\
\hline & & & & & & & & & & Hipo - Hiper $<0,0001$ \\
\hline & & & & & & & & & & Hipo - PDN <0,0001 \\
\hline & & & & & & & & & & Hiper - PDN <0,0001 \\
\hline \multirow{3}{*}{ Lateral } & \multirow{3}{*}{81} & \multirow{3}{*}{91,0} & \multirow{3}{*}{7} & \multirow{3}{*}{7,9} & \multirow{3}{*}{1} & \multirow{3}{*}{1,1} & \multirow{3}{*}{-- } & \multirow{3}{*}{--} & \multirow{3}{*}{$<0,0001$} & N-Hipo <0,0001 \\
\hline & & & & & & & & & & N- Hiper <0,0001 \\
\hline & & & & & & & & & & Hipo-Hiper =0,0771 \\
\hline Anterior & 42 & 93,3 & 3 & 6,7 & -- & -- & -- & -- & $<0,0001$ & N -Hipo <0,0001 \\
\hline \multirow{6}{*}{ Total } & \multirow{6}{*}{846} & \multirow{6}{*}{81,9} & \multirow{6}{*}{136} & \multirow{6}{*}{13,2} & \multirow{6}{*}{42} & \multirow{6}{*}{4,0} & & & & N - Hipo <0,0001 \\
\hline & & & & & & & & & & N - Hiper <0,0001 \\
\hline & & & & & & & 9 & 0.9 & $<00001$ & $\mathrm{~N}-\mathrm{PDN}<0,0001$ \\
\hline & & & & & & & 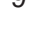 & 0,0 & $<0,0001$ & Hipo -Hiper <0,0001 \\
\hline & & & & & & & & & & Hipo - PDN <0,0001 \\
\hline & & & & & & & & & & Hiper - PDN <0,0001 \\
\hline
\end{tabular}

Nível de significância: $p \leq 0,05$

Legenda: $\mathrm{N}=$ Normorreflexia; Hipo = Hiporreflexia; Hiper = Hiper-reflexia; PDN = Preponderância Direcional do Nistagmo

O canal anterior apresentou prevalência de normorreflexia em comparação com hiporreflexia $(\mathrm{p}<0,0001)$; hiper-reflexia e preponderância direcional do nistagmo pós-calórico não foram identificadas.

Analisando os achados à prova calórica na VPPB, sem considerar o canal comprometido, houve prevalência de normorreflexia em comparação com os demais achados anormais $(\mathrm{p}<0,0001)$; a hiporreflexia foi prevalente sobre a hiper-reflexia $(\mathrm{p}<0,0001)$ e a preponderância direcional do nistagmo póscalórico $(\mathrm{p}<0,0001)$, e a hiper-reflexia foi prevalente sobre a preponderância direcional $(\mathrm{p}<0,0001)$.

A Tabela 3 apresenta o número e a porcentagem de casos com normorreflexia, hiporreflexia, hiper-reflexia ou preponderância direcional do nistagmo pós-calórico, de acordo com o comprometimento unilateral e bilateral do canal posterior na VPPB.

O acometimento unilateral do canal posterior apresentou prevalência de normorreflexia em comparação com os demais achados anormais à prova calórica $(\mathrm{p}<0,0001)$; a hiporreflexia foi prevalente sobre a hiper-reflexia $(\mathrm{p}<0,0001)$ e a preponderância direcional do nistagmo pós-calórico $(p<0,0001)$, e a hiper-reflexia e a preponderância direcional do nistagmo póscalórico não apresentaram diferença significante quanto à prevalência $(p=0,8231)$. Os casos com acometimento bilateral do canal posterior não apresentaram diferença significante quanto à prevalência dos achados da prova calórica ( $\mathrm{p}=0,3194)$.

No acometimento unilateral do canal posterior, a hiporreflexia foi ipsilateral em 92 casos $(88,5 \%)$ e contralateral

Tabela 3. Frequências absolutas, porcentagens e análise comparativa dos achados à prova calórica de acordo com o comprometimento unilateral ou bilateral do canal posterior em 899 pacientes com vertigem posicional paroxística benigna

\begin{tabular}{|c|c|c|c|c|c|c|c|c|c|c|}
\hline \multirow{3}{*}{ Canal } & \multicolumn{8}{|c|}{ Achados à prova calórica } & \multirow{3}{*}{\multicolumn{2}{|c|}{$\begin{array}{l}\text { Qui-quadrado } \\
\text { (valor de } p \text { ) }\end{array}$}} \\
\hline & \multicolumn{2}{|c|}{ Normorreflexia } & \multicolumn{2}{|c|}{ Hiporreflexia } & \multicolumn{2}{|c|}{ Hiper-reflexia } & \multicolumn{2}{|c|}{ PDN } & & \\
\hline & $\mathrm{N}$ & $\%$ & $\mathrm{~N}$ & $\%$ & $\mathrm{~N}$ & $\%$ & $\mathrm{~N}$ & $\%$ & & \\
\hline \multirow{6}{*}{$\begin{array}{l}\text { Posterior } \\
\text { Unilateral }\end{array}$} & \multirow{6}{*}{690} & \multirow{6}{*}{84,7} & \multirow{6}{*}{104} & \multirow{6}{*}{12,8} & \multirow{6}{*}{11} & \multirow{6}{*}{1,4} & \multirow{6}{*}{9} & \multirow{6}{*}{1,1} & \multirow{6}{*}{$<0,0001$} & N - Hipo <0,0001 \\
\hline & & & & & & & & & & N - Hiper $<0,0001$ \\
\hline & & & & & & & & & & $\mathrm{N}-\mathrm{PDN}<0,0001$ \\
\hline & & & & & & & & & & Hipo -Hiper $<0,0001$ \\
\hline & & & & & & & & & & Hipo - PDN <0,0001 \\
\hline & & & & & & & & & & Hiper - PDN =0,8231 \\
\hline $\begin{array}{l}\text { Posterior } \\
\text { Bilateral }\end{array}$ & 33 & 38,8 & 22 & 25,9 & 30 & 35,3 & -- & -- & 0,3194 & -- \\
\hline Total & 723 & & 126 & & 41 & & 9 & & & \\
\hline
\end{tabular}

Nível de significância: $p \leq 0,05$

Legenda: $\mathrm{N}=$ Normorreflexia; Hipo = Hiporreflexia; Hiper = Hiper-reflexia; PDN = Preponderância Direcional do Nistagmo 
em 12 casos (11,5\%); a hiper-reflexia foi bilateral nos 11 casos $(100,0 \%)$, e a preponderância direcional do nistagmo pós-calórico foi contralateral ao labirinto comprometido nos nove casos $(100,0 \%)$. No acometimento bilateral do canal posterior, a hiporreflexia foi bilateral em 18 casos $(21,2 \%) \mathrm{e}$ unilateral em quatro $(4,7 \%)$ e a hiper-reflexia foi bilateral nos 30 casos $(100,0 \%)$.

No acometimento do canal lateral, a hiporreflexia foi ipsilateral nos sete casos $(100,0 \%)$ e a hiper-reflexia foi bilateral em um caso $(100,0 \%)$.

No acometimento do canal anterior, a hiporreflexia foi ipsilateral nos três casos $(100,0 \%)$.

\section{DISCUSSÃO}

Analisando os prontuários de 1033 pacientes com VPPB, verificamos que o comprometimento do canal semicircular posterior foi significativamente mais frequente $(87,0 \%$ dos casos) do que o dos canais lateral ( $8,6 \%$ dos casos) e anterior (4,4\% dos casos). Nossos achados estão de acordo com a opinião de alguns autores, de que a VPPB acomete com maior frequência o canal semicircular posterior, mas também pode envolver o canal lateral e o canal anterior ${ }^{(5-7)}$. Nossos achados e os destes autores diferem da prevalência de acometimento do canal lateral observada em 16,4\% dos casos por outro estudo ${ }^{(8)}$.

Nesta pesquisa, a VPPB unilateral $(91,8 \%$ dos casos) foi mais comum do que a bilateral $(8,2 \%)$, à semelhança dos achados de outros autores que também observaram o acometimento unilateral mais frequente $(88,2 \%)$ do que o bilateral $(11,9 \%)^{(2)}$.

Independentemente do canal comprometido na VPPB, evidenciamos que normorreflexia ( $81,9 \%$ dos casos) foi mais frequente do que os outros sinais à prova calórica. Os nossos achados são concordantes com os de outros autores que referiram ser a prova calórica usualmente normal na maioria dos pacientes com VPPB ${ }^{(13-14)}$, mas diferem da prevalência da normorreflexia encontrada em outros estudos, de 47,6\% e $57,2 \%$ dos $\operatorname{casos}^{(6-7)}$.

No que diz respeito aos nossos achados anormais à prova calórica, independentemente do canal comprometido, hiporreflexia $(13,2 \%)$ prevaleceu sobre hiper-reflexia $(4,0 \%)$ e preponderância direcional do nistagmo pós-calórico $(0,9 \%)$, e hiper-reflexia foi mais comum do que a preponderância direcional. Diferentes prevalências dos achados anormais do nistagmo pós-calórico foram mencionadas na literatura: hiporreflexia em 16,6\% dos $\operatorname{casos}^{(15)}$; hiporreflexia em $27,0 \%$ dos casos, preponderância direcional em 17,2\% e preponderância direcional concomitante com hiporreflexia em 8,2\% hiporreflexia em 22,0\% dos casos, preponderância direcional em $13,7 \%$ e hiporreflexia concomitante com preponderância direcional em $7,1 \%{ }^{(6)}$.

Na prova calórica dos casos de VPPB de canal posterior, a normorreflexia (80,4\% dos casos) foi mais frequente do que os demais achados anormais, concordando com os achados de outros autores $^{(16)}$.

No que diz respeito aos nossos achados anormais à prova calórica dos casos de VPPB de canal posterior, hiporreflexia
$(14,0 \%)$ predominou sobre hiper-reflexia $(4,6 \%)$ e preponderância direcional $(1,0 \%)$; hiper-reflexia foi mais frequente do que preponderância direcional. A literatura mostra ampla variação de resultados de autor para autor: hiporreflexia em $21,1 \%$ dos casos e preponderância direcional em 36,8\%(13); hiporreflexia em cerca de $39 \%$ dos casos e preponderância direcional em cerca de $8 \%{ }^{(16)}$; hiporreflexia em 26,4\% dos casos, preponderância direcional em 18,2\% e hiporreflexia concomitante com preponderância direcional em 5,5\% ${ }^{(7)}$.

$\mathrm{Na}$ VPPB de canal posterior unilateral, normorreflexia $(84,7 \%)$ foi mais frequente do que os outros achados à prova calórica, dado semelhante ao encontrado por alguns autores ${ }^{(17)}$. No entanto, outro autor observou a hiporreflexia em 45,0\% dos casos, superando em prevalência a normorreflexia encontrada em nosso estudo que ocorreu em $30,0 \%{ }^{(18)}$.

No que diz respeito aos nossos achados anormais à prova calórica dos casos de VPPB de canal posterior unilateral, hiporreflexia $(12,8 \%)$ foi mais frequente do que hiper-reflexia $(1,4 \%)$ e preponderância direcional $(1,1 \%)$; hiper-reflexia e preponderância direcional não apresentaram diferença significante em prevalência. A hiper-reflexia foi bilateral e a preponderância direcional foi contralateral ao labirinto comprometido em todos os casos $(100,0 \%)$ em que ocorreram. No total de nossos casos com hiporreflexia, este achado foi ipsilateral $(88,5 \%)$ ou contralateral $(11,5 \%)$. A literatura mostra ampla variação nos resultados de diversos autores: hiporreflexia ipsilateral em $17,0 \%$ dos casos, hiporreflexia contralateral em $6,0 \%$, hiporreflexia bilateral em $8,0 \%$ e preponderância direcional em $8,0 \%{ }^{(17)}$; hiporreflexia ipsilateral em $8,2 \%$ dos casos e hiporreflexia contralateral em $5,5 \%{ }^{(7)}$; hiporreflexia ipsilateral em 45,0\% dos casos, hiporreflexia contralateral em $15,0 \%$ e preponderância direcional em $5,0 \%{ }^{(18)}$.

$\mathrm{Na}$ VPPB de canal posterior bilateral, não ocorreu diferença significante quanto à prevalência dos achados à prova calórica: normorreflexia $(38,8 \%)$, hiporreflexia unilateral $(4,7 \%)$ ou bilateral $(21,2 \%)$ e hiper-reflexia bilateral $(35,3 \%)$. Não encontramos achados na literatura para comparação.

À prova calórica nos nossos casos de VPPB de canal lateral, a normorreflexia $(91,0 \%)$ foi mais frequente do que hiporreflexia $(7,9 \%)$ e hiper-reflexia $(1,1 \%)$ e a hiporreflexia apresentou tendência de prevalência sobre a hiper-reflexia. A hiporreflexia foi ipsilateral e a hiper-reflexia foi bilateral em todos os casos $(100,0 \%)$ em que ocorreram. Não ocorreu preponderância direcional do nistagmo pós-calórico. A literatura mostra ampla variação à comparação dos resultados de vários autores. A hiporreflexia ipsilateral foi observada 33,3\% dos $\operatorname{casos}^{(19)}$, em $30,8 \%{ }^{(15)}$, em um paciente ${ }^{(21)}$, em cerca de $30,0 \%{ }^{(22)}$ e em $50,0 \%{ }^{(23)}$. Encontraram hiporreflexia ipsilateral em $0,8 \%$ dos casos e bilateral em $0,8 \%$, preponderância direcional em $0,8 \%$ e hiporreflexia ipsilateral concomitante com preponderância direcional em $3,2 \%^{(7)}$.

Na prova calórica nos casos de VPPB de canal anterior, normorreflexia $(93,3 \%)$ predominou sobre hiporreflexia $(6,7 \%)$; hiper-reflexia e preponderância direcional do nistagmo póscalórico não foram encontradas. A hiporreflexia foi ipsilateral em todos os casos em que ocorreu. Contrastando com outro estudo que identificou hiporreflexia ipsilateral em $0,8 \%$ dos casos e bilateral em $0,8 \%{ }^{(7)}$. 
Os nossos achados na VPPB concordam com os de outros autores quanto ao comprometimento do canal semicircular posterior ser mais frequente do que o dos canais lateral e anterior e quanto a VPPB unilateral ser mais comum do que a bilateral.

Comparando os nossos dados com as informações da literatura pertinente, verificamos uma ampla variação da prevalência dos achados à prova calórica na VPPB dos canais: posterior, lateral e anterior de autor para autor, exigindo novos estudos para melhor elucidação. A análise da associação de achados da prova calórica e da pesquisa do nistagmo de posicionamento e posicional contribui para a caracterização da VPPB.

A identificação de hiporreflexia do nistagmo pós-calórico do mesmo lado ou do lado oposto da VPPB de canal posterior ou anterior ocorreu em 13,7\% dos casos (129/944), caracterizando o envolvimento concomitante de canal lateral. A hiporreflexia pode ser um achado ocasionado por uma lesão que já está compensada há muito tempo ou pode sugerir investigação adicional para excluir um schwannoma vestibular ou outro tumor do ângulo pontocerebelar. Excluída estas possibilidades, a hiporreflexia vestibular unilateral pode ser causada pela própria VPPB, propiciando sintomas que necessitem de procedimento complementar de reabilitação vestibular, além da manobra específica para o canal comprometido na VPPB.

A VPPB pode surgir em decorrência de diversas afecções vestibulares como, por exemplo, hidropisia endolinfática, migrânea vestibular, neurite vestibular, labirintopatias vasculares, metabólicas, traumáticas ou afecções do sistema nervoso central, entre outras. Nestas situações, a prova calórica pode contribuir para identificar a topografia periférica e/ou central da disfunção vestibular relacionada com o aparecimento, recorrência ou persistência da VPPB.

A prova calórica não tem o objetivo de identificar a etiologia dos distúrbios vestibulares, mas pode evidenciar se há ou não comprometimento funcional de um ou dos dois labirintos e auxiliar na obtenção de um diagnóstico mais acurado e na formulação de uma estratégia terapêutica adequada.

\section{CONCLUSÃO}

Avaliando os achados na prova calórica de pacientes com VPPB, concluímos que: no comprometimento do canal posterior, ocorrem normorreflexia, hiporreflexia contralateral à VPPB, hiper-reflexia e preponderância direcional do nistagmo pós-calórico, em ordem decrescente de prevalência; no comprometimento do canal lateral, normorreflexia prevalece sobre hiporreflexia unilateral ipsilateral à VPPB e hiper-reflexia bilateral; a hiporreflexia tende a prevalecer sobre hiper-reflexia, e no comprometimento do canal anterior, normorreflexia prevalece sobre hiporreflexia unilateral ipsilateral à VPPB.

\begin{abstract}
Purpose: To characterize caloric test results in benign paroxysmal positional vertigo patients, according to the damaged semicircular canal. Methods: The data of 1033 patients submitted to Dix-Hallpike testing, positional nystagmus and electronystagmography were analyzed. Caloric test results were compared to the damaged semicircular canals and were submitted to statistical analysis. Results: In cases with posterior canal damage, there was prevalence of normal responses compared to abnormal results ( $\mathrm{p}<0.0001)$; hypo activity was more prevalent than hyperactivity $(\mathrm{p}<0.0001)$ and directional preponderance $(\mathrm{p}<0.0001)$, and hyperactivity more prevalent than directional preponderance $(\mathrm{p}<0.0001)$. In cases with lateral canal damage, normal responses were more prevalent than hypo activity $(\mathrm{p}<0.0001)$ and hyperactivity $(\mathrm{p}<0.0001)$; there was a tendency of prevalence of hypo activity over hyperactivity $(\mathrm{p}=0.0771)$, and directional preponderance was not observed. In cases with anterior canal damage, normal responses were more prevalent than hypo activity $(\mathrm{p}<0.0001)$; hyperactivity and directional preponderance were not observed. Conclusion: In the caloric test of benign positional paroxysmal vertigo patients, normal responses, hypo activity, hyperactivity or directional preponderance of post-caloric nystagmus occur in decreasing order of prevalence when the posterior canal is damaged; normal responses are more prevalent than hypoactive or hyperactive caloric responses, and there is a tendency of prevalence of hypoactive over hyperactive caloric responses when the lateral canal is damaged; normal responses are more prevalent than hypoactive caloric responses when the anterior canal is damaged.
\end{abstract}

Keywords: Ear; Inner; Semicircular canals; Dizziness; Vertigo; Electronystagmography; Caloric tests/methods

\title{
REFERÊNCIAS
}

1. Ganança MM, Caovilla HH. Desequilíbrio e reequilíbrio. In: Ganança MM, editor. Vertigem tem cura?: o que aprendemos nestes últimos 30 anos. São Paulo: Lemos Editorial; 1998. p.13-9.

2. Ganança MM, Caovilla HH, Munhoz MSL, Silva MLG, Ganança FF, Ganança CF. Vertigem posicional paroxística benigna. Rev Bras Med Otorrinolaringol. 2000;7(3):66-72.

3. Schuknecht HF. Cupulolithiasis. Arch Otolaryngol. 1969;90(6):76578.
4. Hall SF, Ruby RR, McClure JA. The mechanics of benign paroxysmal vertigo. J Otolaryngol. 1979;8(2):151-8.

5. Parnes LS, Agrawal SK, Atlas J. Diagnosis and management of benign paroxysmal positional vertigo (BPPV). CMAJ. 2003;169(7):681-93. Review.

6. Korres SG, Balatsouras DG, Ferekidis E. Electronystagmographic findings in benign paroxysmal positional vertigo. Ann Otol Rhinol Laryngol. 2004;113(4):313-8. 
7. Korres S, Balatsouras DG, Kaberos A, Economou C, Kandiloros D, Ferekidis E. Occurrence of semicircular canal involvement in benign paroxysmal positional vertigo. Otol Neurotol. 2002;23(6):926-32.

8. De la Meilleure G, Dehaene I, Depondt M, Damman W, Crevits L, Vanhooren G. Benign paroxysmal positional vertigo of the horizontal canal. J Neurol Neurosurg Psychiatry. 1996;60(1):68-71.

9. Dix MR, Hallpike CS. The pathology, symptomatology and diagnosis of certain common disorders of the vestibular system. Ann Otol Rhinol Laryngol. 1952;61(4):987-1016.

10. Herdman SJ. Reabilitação vestibular. Barueri: Manole; 2002.

11. Caovilla HH, Ganança MM, Munhoz MS, Silva MG. Equilibriometria clínica. São Paulo: Atheneu; 1999. 158p.

12. Ganança MM, Caovilla HH, Freitas-Ganança F, Munhoz MSL, Silva MLG. Vertigem posicional paroxística benigna. In: Silva MLG, Munhoz MSL, Ganança MM, Caovilla HH, editores. Quadros clínicos otoneurológicos mais comuns. São Paulo: Atheneu; 2000. p. 9-19. [Série Otoneurológica, 3].

13. Toupet M, Lasserre MH, Laccourreye H. Nystagmus et vertige paroxystiques bénins. Ann Otolaryngol Chir Cervicofac. 1982;99(12):7-14.

14. Bachor E, Wright CG, Karmody CS. The incidence and distribution of cupular deposits in the pediatric vestibular labyrinth. Laryngoscope. 2002;112(1):147-51.

15. Wong ML, Rauch-Mahoney C, Catterall NS, Voorhees RL, Maclean JB. Comparación de trés pruebas de función vestibular. Ann Otorrinolaringol Mex. 1993:38(2):73-7.

16. Baloh RW, Honrubia V, Jacobson K. Benign positional vertigo: clinical and oculographic features in 240 cases. Neurology. 1987;37(3):371-8.

17. Dumas G, Charachon R, Ghozali S. [Benign paroxysmal positional vertigo. Apropos of 51 cases]. Ann Otolaryngol Chir Cervicofac. 1994;111(6):301-13. French.
18. Amorim M, Gapo C, Costa S, Paiva S, Neves JC, Tomé P, Sofia JC, Paiva A. Vertigem posicional paroxística benigna e hipovalência labiríntica [Internet]. Coimbra: Associação Portuguesa de Otoneurologia. [cited 2006 Ago 17]. Disponível em: http://www.otoneuro.pt/images/stories/ forum/textosnovos/forum19.pdf

19. Pagnini P, Nuti D, Vannucchi P. Benign paroxysmal vertigo of the horizontal canal. ORL J Otorhinolaryngol Relat Spec. 1989;51(3):16170.

20. Baloh RW, Jacobson K, Honrubia V. Horizontal semicircular canal variant of benign positional vertigo. Neurology. 1993;43(12):2542-9. Comment in: Neurology. 1994;44(11):2213-4.

21. Strupp M, Brandt T, Steddin S. Horizontal canal benign paroxysmal positioning vertigo: reversible ipsilateral caloric hypoexcitability caused by canalolithiasis? Neurology. 1995;45(11):2072-6.

22. Brandt T. Vertigo: its multisensory syndromes. 2nd ed. London: Springer; 1999. 503p.

23. Di Girolamo S, Ottaviani F, Scarano E, Picciotti P, Di Nardo W. Postural control in horizontal benign paroxysmal positional vertigo. Eur Arch Otorhinolaryngol. 2000;257(7):372-5.

24. Koga KA, Resende BA, Mor R. Estudo da prevalência de tontura/ vertigens e das alterações vestibulares relacionadas à mudança de posição de cabeça por meio da vectoeletronistagmografia computadorizada. Rev CEFAC. 2004;6(2):197-202.

25. Roberts RA, Gans RE, Kastner AH, Lister JJ. Prevalence of vestibulopathy in benign paroxysmal positional vertigo patients with and without prior otologic history. Int J Audiology. 2005;44(4):191-6.

26. Ganança MM, Caovilla HH, Munhoz MSL, Silva MGL, Frazza MM. As etapas da equilibriometria. In: Caovilla HH, Ganança MM, Munhoz MS, Silva MG. Equilibriometria clínica. São Paulo: Atheneu; 1999. p.41-5. 